\title{
Adaptive pulse-shaped OFDM with application to in-home power line communications
}

\author{
Salvatore D’Alessandro • Andrea M. Tonello • \\ Lutz Lampe
}

Published online: 20 January 2011

(C) The Author(s) 2010. This article is published with open access at Springerlink.com

\begin{abstract}
We propose an adaptive pulse-shaped orthogonal frequency division multiplexing (OFDM) scheme where the overhead and active sub-channels are adapted to jointly maximize capacity and fulfill the notching mask for coexistence with other devices operating in the same spectrum. The overhead includes the guard interval to partly compensate the channel dispersion, and the roll-off factor used by the shaping window. We show that significant gains are obtained compared to the case of using a fixed guard interval and roll-off factor. To simplify the implementation complexity we also propose to limit the amount of adaptation by deploying a finite set of overhead parameters. Numerical results are reported for typical wideband power line communication indoor channels.
\end{abstract}

Keywords OFDM · Pulse-shaped OFDM · Power line communications · In-home networking

Part of this work has been presented at the 2008 IFIP Wireless Days Conference. The work of S. D'Alessandro and A.M. Tonello has been partially supported by the European Community's Seventh Framework Programme FP7/2007-2013 under grant agreement $\mathrm{n}^{\circ}$ 213311, project OMEGA-Home Gigabit Networks. The work of L. Lampe has been supported by the National Sciences and Engineering Research Council of Canada (NSERC).

S. D’Alessandro · A.M. Tonello $(\bowtie)$

Dipartimento di Ingegneria Elettrica, Gestionale e Meccanica, Universitá degli Studi di Udine, via delle Scienze 208, 33100

Udine, Italy

e-mail: tonello@uniud.it

S. D'Alessandro

e-mail: salvatore.dalessandro@uniud.it

L. Lampe

Department of Electrical and Computer Engineering, University

of British Columbia, Vancouver, BC, V6T1Z4, Canada

e-mail: lampe@ece.ubc.ca

\section{Introduction}

Multicarrier (MC) modulation is becoming the most popular modulation technique for transmission over wide band channels that exhibit frequency selectivity both in wireless and wireline applications, e.g., in power line communications (PLC) as considered in this paper. Two important examples of MC modulation are orthogonal frequency division multiplexing (OFDM) and pulse-shaped OFDM (PS-OFDM) [1], [2, Chap. 5]. The former scheme uses a rectangular pulse shape, while the latter uses a Nyquist window to provide better sub-channel confinement. PS-OFDM is employed in the FFT-OFDM physical layer modality of the IEEE 1901 standard which operates in the band $2-30 \mathrm{MHz}$ and grants backward compatibility with the HomePlug AV (HPAV) standard [3, 4], [2, Chap. 7]. This frequency band contains also subbands dedicated to other communication systems, as for example amateur radio. Hence, in order to allow coexistence a number of sub-channels are switched off such that the transmitted signal occupies a certain spectrum with notches. Since PS-OFDM has a better sub-channel spectral containment it is capable of respecting the mask by switching off a lower number of sub-channels than OFDM.

An attractive feature of both OFDM and PS-OFDM is that with the use of the guard interval (GI) the dispersive channel can be orthogonalized such that simple one-tap equalization per sub-channel can be used. The conventional choice is to use a GI of $\mu$ samples that is longer than the channel duration [5]. Unfortunately, this advantage has to be paid in terms of both a transmission rate loss and a signalto-noise ratio penalty [6].

Since PS-OFDM uses a pulse shaping window with a roll-off (RO) equal to $\alpha$ samples, it introduces an overhead (OH) equal to $\alpha+\mu$ samples which yields a loss in transmission rate by a factor equal to $M /(M+\mu+\alpha)$ where $M$ is the number of sub-channels. 
In this paper we address the problem of optimizing the system parameters $\alpha$ and $\mu$. The optimization criterion is based on a capacity expression. We note that the guard interval does not necessarily need to be as long as the channel impulse response to maximize capacity. In fact, in [7] we have shown that when considering OFDM the optimal guard interval is shorter than the channel length. Furthermore, we have found that significant gains can be obtained adapting the GI to the channel realization w.r.t. the choice of using a single value of GI.

In this paper we extend the analysis done in [7] for OFDM to the case of PS-OFDM and we also consider the notching problem. We propose to adapt the $\mathrm{OH}$ parameters $\alpha$ and $\mu$ to the channel realization such that we jointly maximize capacity and fulfill the notching mask for coexistence with other devices operating in the same spectrum. This realizes an adaptive PS-OFDM scheme.

To lower the computational cost, we also propose to limit the amount of adaptation, i.e., to use a finite set of $\mathrm{OH}$ parameters. The $\mathrm{OH}$ parameters can be computed from the analysis of their distribution function in statistically representative PLC channels. Since a significant change of the $\mathrm{OH}$ parameters is determined by a significant change in the signal-to-noise ratio ( $\mathrm{SNR}$ ), we consider the design of the $\mathrm{OH}$ parameters for a low, a medium, and a high SNR scenario assuming a certain target notching mask. Thus, the adaptation is limited to the computation of the SNR at the receiver, followed by feedback to the transmitter of the best $\mathrm{OH}$ parameters for such an SNR.

This paper is organized as follows. In Sect. 2, we describe the system model, the OFDM and the PS-OFDM transmission schemes. In Sect. 3, adaptive PS-OFDM is considered and the problem of optimizing the system parameters is discussed. Limited adaptation is proposed in Sect. 4. The numerical results are reported in Sect. 5. Finally, conclusions are offered in Sect. 6.

\section{System model}

OFDM and Pulse-Shaped OFDM [1], [2, Chap. 5] are two important examples of filter bank modulation (FBM). In FBM a high rate data signal is split into $M$ parallel data signals $a^{(k)}(\ell N), k=\{0, \ldots, M-1\}$, where $N$ denotes the normalized sub-channel symbol period. Each data signal is interpolated by a factor $N$, filtered with a prototype pulse $g(n)$, and exponentially modulated to the $k$-th (normalized) sub-carrier frequency $f_{k}=k / M$. Therefore, the discretetime multicarrier signal can be written as the output of a synthesis filter bank (FB) as follows

$x(n)=\sum_{k=0}^{M-1} \sum_{\ell \in \mathbb{Z}} a^{(k)}(\ell N) g(n-\ell N) e^{j \frac{2 \pi k n}{M}}$.
The data symbol $a^{(k)}(\ell N)$ is selected from a quadrature amplitude modulation (QAM) constellation. For each subchannel $k$, the constellation order is chosen according to the channel condition, i.e., bit-loading is employed to approach the channel capacity [8].

We define the $\mathrm{OH}$ factor $\beta=N-M$. If it is larger than zero, the FB will not be critically sampled. For instance, $\beta$ equals the guard interval length in OFDM.

We assume that the signal (1) is transmitted over a channel that has an equivalent discrete time complex impulse response

$g_{C H}(n)=\sum_{p=0}^{v-1} \alpha_{p} \delta(n-p)$,

where $\alpha_{p}$ denote the complex channel gains, and $\delta(n)=1$ for $n=0$, and zero otherwise.

The received signal

$y(n)=x(n) * g_{C H}(n)+w(n)$,

where $w(n)$ is the additive noise and $*$ denotes convolution, is processed with an analysis FB having prototype pulse $h(n)$. The FB outputs are sampled at rate $1 / N$ to obtain

$$
\begin{aligned}
z^{(k)}(\ell N+\Delta)= & \sum_{n \in \mathbb{Z}} y(n) h(\ell N+\Delta-n) e^{-j \frac{2 \pi k n}{M}} \\
= & H^{(k)}(\mu, \alpha, \Delta) a^{(k)}(\ell N) \\
& +I^{(k)}(\mu, \alpha, \ell N+\Delta) \\
& +\eta^{(k)}(\ell N+\Delta)
\end{aligned}
$$

where $-\mu \leq \Delta \leq \mu$ is the synchronization time phase. Furthermore, $H^{(k)}(\mu, \alpha, \Delta)$ is the amplitude of the data of interest, $I^{(k)}(\mu, \alpha, \ell N+\Delta)$ is the overall interference that includes the inter-symbol interference (ISI) and the intercarrier interference (ICI). ISI and ICI occur when transmitting over a frequency-selective channel with a guard interval $\mu<v-1$. As discussed in the following, the factor $H^{(k)}(\mu, \alpha, \Delta)$ and the interference term $I^{(k)}(\mu, \alpha, \ell N+\Delta)$ are functions of the pulse roll-off $\alpha$ and the guard interval $\mu$. Finally, $\eta^{(k)}(\ell N+\Delta)$ represents the noise contribution.

\subsection{OFDM}

In OFDM the synthesis and the analysis pulses are rectangular windows respectively defined as

$g(n)=\operatorname{rect}\left(\frac{n}{N}\right), \quad h(n)=\operatorname{rect}\left(-\frac{n+\mu}{M}\right)$,

where $\operatorname{rect}(n / A)=1$ for $n=0, \ldots, A-1$ and zero otherwise. The synthesis window has duration $N=M+\mu$ samples. Therefore, the $\mathrm{OH}$ factor is equal to $\beta=\mu$ samples. 
The efficient implementation of OFDM at the transmitter comprises the following steps: compute an $M$-point inverse discrete Fourier transform (IDFT) of the block of data symbols $a^{(k)}(\ell N)$ for $k=0, \ldots, M-1$; then, add a guard interval in the form of a cyclic prefix (CP) that equals the last $\mu$ IDFT output coefficients. At the receiver: acquire symbol synchronization, discard the $\mathrm{CP}$, and apply an $M$-point DFT on the remaining $M$ samples. It should be noted that according to the definitions in (4) and (5), the DFT window starts $\pm \Delta$ samples after (before) the cyclic prefix.

As it is well known, if the length of the channel impulse response does not exceed the $\mathrm{CP}$ length by more than one, i.e., $\mu \geq v-1$, the system will be orthogonal such that the received symbol will be neither affected by ISI nor by ICI [5]. Nevertheless, this advantage has to be paid in terms of an SNR penalty and a raw transmission rate loss that is equal to $M /(M+\mu)$.

Another drawback with OFDM is that the sub-channels have a sinc frequency response that decays as $1 / f$ and has the first side lobe only $13 \mathrm{~dB}$ down compared to the main lobe. This translates in poor sub-channel confinement, and consequently, in a large transmission rate penalty when notching is required to enable coexistence with other systems. In fact, to fulfill stringent notching masks a large number of sub-channels has to be switched off.

\subsection{Pulse-shaped OFDM}

To overcome the poor sub-channel frequency confinement, a window better than the rectangular window can be deployed. The scheme is referred to as PS-OFDM [9]. In PS-OFDM the synthesis prototype pulse is a Nyquist window. A common choice is to use a raised cosine window with roll-off $\alpha$ samples [9]. It can be defined as follows

$g(n)=\left\{\begin{aligned} & \frac{1}{2}+\frac{1}{2} \cos \left(\frac{\pi}{\alpha}\left(\left|n-n_{1}\right|-n_{2}\right)\right) \\ & n \in[0, \alpha] \cup[N, N+\alpha], \\ & 1n \in] \alpha, N[, \\ & 0 \text { otherwise, }\end{aligned}\right.$

with $n_{1}=(N+\alpha) / 2$ and $n_{2}=n_{1}-\alpha$. With $\alpha=0$ we obtain the conventional OFDM scheme.

In PS-OFDM the symbol period increases by $\alpha$ samples w.r.t. OFDM assuming that we still deploy a guard interval of length $\mu$ samples, i.e., $N=M+\mu+\alpha$. Consequently, the $\mathrm{OH}$ factor is equal to $\beta=\mu+\alpha$, and the transmission rate loss is equal to $M /(M+\mu+\alpha)$ that turns out to be higher than in OFDM.

It can be shown that the efficient implementation (Fig. 1) of PS-OFDM comprises an $M$-point IDFT, followed by a CP extension of $\mu+\alpha$ samples, and a cyclic postfix extension of $\alpha$ samples. Then, a windowing with a window of duration $N+\alpha$ samples is applied. Finally, the signal to be

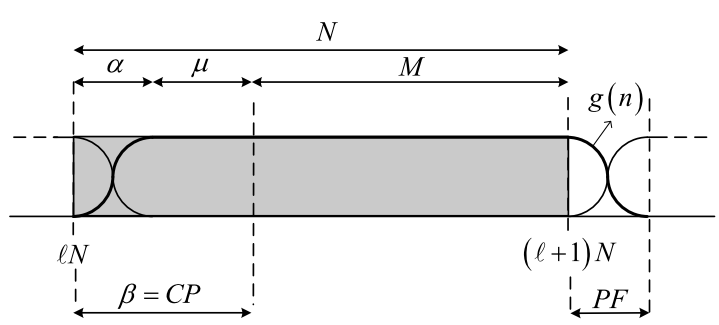

Fig. 1 Generation of the $\ell$-th symbol in PS-OFDM

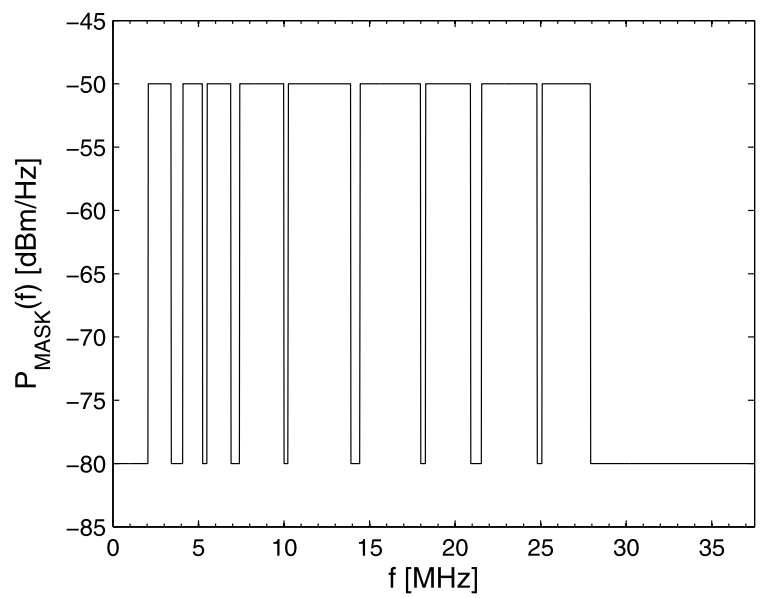

Fig. 2 Power spectral density mask for the transmitted signal. With a carrier spacing of $24.414 \mathrm{kHz}$ (as in HPAV), the number of sub-channel in $0-37.5 \mathrm{MHz}$ is equal to 1536 , among which 619 are notched

transmitted is obtained by overlapping and adding the first $\alpha$ samples of each symbol with the last $\alpha$ samples of the preceding symbol [2, Chap. 5].

In PS-OFDM, the analysis pulse is a rectangular window of duration $M$ samples that is defined as

$h(n)=\operatorname{rect}\left(-\frac{n+\beta}{M}\right)$.

Again, if $\mu \geq v-1$ the system will be orthogonal.

\subsection{Notching}

State-of-the-art broadband PLC systems operate in the band $2-30 \mathrm{MHz}$ and optionally even above $30 \mathrm{MHz}$ [2]. This frequency band contains also sub-bands dedicated to other communication systems, as for example amateur radio. Furthermore, above $30 \mathrm{MHz}$ the limits for radiated emissions are much more stringent than those in the band below $30 \mathrm{MHz}$. Hence, in order to allow the coexistence with other systems, the PLC modem has to be capable of respecting a flexible notching mask. In Fig. 2, we show a representative example of power spectral density (PSD) mask which is similar to the one employed in the HPAV system [4]. The PSD of the transmitted signal is set at $-50 \mathrm{dBm} / \mathrm{Hz}$, while the notched portions of the spectrum are set at $-80 \mathrm{dBm} / \mathrm{Hz}$. 

[10]

The transmitted signal PSD can be computed as follows

$$
P_{x}(f)=\frac{P_{a}}{N T} \sum_{k \in K_{O N}(\alpha, \mu)}\left|G\left(f-f_{k}\right)\right|^{2}, \quad f \in[0, B],
$$

if we assume the data symbols to be i.i.d. with zero mean and power $P_{a}$. Further, $T$ is the sampling period in the system, $B=1 / T$ is the system transmission band, $G(f)$ is the discrete time Fourier transform of $g(n)$, and $K_{O N}(\alpha, \mu)$ is the set of tones that are switched on. It is a function of the $\mathrm{OH}$ parameters $\alpha$ and $\mu$, and for a certain choice of them, the set of active tones is determined to satisfy a target PSD mask $P_{\text {MASK }}(f)$, as follows

$$
P_{x}(f) \leq P_{\text {MASK }}(f), \quad f \in[0, B]
$$

Since PS-OFDM has a better sub-channel spectral containment, it is capable of respecting the mask by switching off a smaller number of sub-channels than OFDM. In other words, the set of used sub-channels in PS-OFDM has a cardinality that is greater than the one of OFDM when notching has to be taken into account. Therefore, although PS-OFDM requires a higher $\mathrm{OH}$ factor than OFDM, it may be able to transmit at a higher rate when notching has to be taken into account.

In the next section we propose a system design criterion based on the maximization of the capacity. That is, we propose to choose the $\mathrm{OH}$ factor such that maximum capacity is achieved. Furthermore, since the channel condition can change we propose to adapt the system parameters.

\section{Adaptive pulse-shaped OFDM}

Power line networks were not designed as a communication medium. Within a network, wires of different length and/or section are used. Furthermore, different loads are plugged into the network outlets. In general, these loads have an impedance that is time variant. This causes the channel between two nodes to vary with time and to depend on the pair of nodes considered. Therefore, it is reasonable to adapt the system $\mathrm{OH}$, namely the roll-off factor and the guard interval, to the specific channel realization. Furthermore, the notching mask is not necessarily static. That is, adaptive notching can be implemented to avoid parts of the spectrum in which the presence of another signal has been detected such as an RF signal captured by the PLC network. This realizes a form of cognitive PLC system.

In the following, Sect. 3.1 describes a criterion to adapt the $\mathrm{OH}$ based on the capacity maximization. As it will be explained, this method is computationally complex. Therefore, we also propose a simpler suboptimal method that limits the amount of adaptation in Sect. 3.2.

\subsection{Channel capacity criterion}

As shown in (4), the FB sub-channel output may experience not only noise, but also ISI and ICI. If the guard interval is sufficiently long $(\mu \geq v-1)$, then both OFDM and PSOFDM are orthogonal, and neither ISI nor ICI is present. However, if the guard interval is too short, interference will occur. We can express the signal-over-interference-plusnoise ratio (SINR) in the $k$-th sub-channel output (4), for a certain channel realization, as follows

$\operatorname{SINR}^{(k)}(\mu, \alpha, \Delta)=\frac{P_{U}^{(k)}(\mu, \alpha, \Delta)}{P_{\eta}^{(k)}+P_{I}^{(k)}(\mu, \alpha, \Delta)}$,

with

$$
\begin{aligned}
& P_{U}^{(k)}(\mu, \alpha, \Delta)=\left|H^{(k)}(\mu, \alpha, \Delta)\right|^{2} E\left[\left|a^{(k)}(\ell N)\right|^{2}\right], \\
& P_{I}^{(k)}(\mu, \alpha, \Delta)=E\left[\left|I^{(k)}(\mu, \alpha, \ell N+\Delta)\right|^{2}\right], \\
& P_{\eta}^{(k)}=E\left[\left|\eta^{(k)}(\ell N+\Delta)\right|^{2}\right] .
\end{aligned}
$$

The relations (11)-(13), respectively, denote the useful, the interference, and the noise power terms on sub-channel $k$, and $E[\cdot]$ is the expectation operator. A detailed derivation of the power terms for OFDM can be found in [7]. In the Appendix we present the expressions for PS-OFDM.

In order to evaluate the impact of the $\mathrm{OH}$ length on the PS-OFDM system performance we evaluate capacity assuming single tap sub-channel equalization and using the formula for parallel Gaussian channels, i.e., the noise and the input signals are independent and Gaussian distributed such that the interference signals from an insufficient $\mathrm{OH}$ are also Gaussian. We further assume a PSD constraint such that the power is uniformly distributed across the used subchannels (see Fig. 2).

Therefore, if $K_{O N}(\mu, \alpha)$ is the set of active tones indices that allows fulfilling the transmission mask, the capacity in $\mathrm{bit} / \mathrm{s}$ is

$$
\begin{aligned}
& C(\mu, \alpha, \Delta) \\
& =\frac{1}{(M+\beta) T} \\
& \quad \times \sum_{k \in K_{O N}(\mu, \alpha)} \log _{2}\left(1+\frac{\operatorname{SINR}^{(k)}(\mu, \alpha, \Delta)}{\Gamma}\right),
\end{aligned}
$$

where $\Gamma$ represents a gap factor to take into account practical implementation constraints [8, 11]. It should be noted that the set $K_{O N}(\mu, \alpha)$ depends on the $\mathrm{OH}$ parameters. As it will be shown in the numerical results section, for a fixed number of sub-channels $M$, as the $\mathrm{OH}$ increases both the cardinality of the set of active sub-channel $\left|K_{O N}\right|$ and the SINR increase, whereas the raw transmission rate decreases. Furthermore, we point out that the capacity is a function not 
only of the $\mathrm{OH}$ but also of the synchronization phase $\Delta$. Therefore, the optimal $\mathrm{OH}$ and synchronization phase can be chosen so that (14) is maximized as follows

$\left(\mu_{O P T}, \alpha_{O P T}, \Delta_{O P T}\right)=\underset{\mu, \alpha, \Delta}{\arg \max }\{C(\mu, \alpha, \Delta)\}$.

Finding the optimal solution of (15) is a complex problem since it requires the full search of the three parameters $(\mu, \alpha, \Delta)$. To simplify it, we propose to first set the synchronization phase with a simple criterion. That is, the capacity criterion is used only for the search of the pair of parameters $(\alpha, \mu)$ as follows

$\left(\mu_{O P T}, \alpha_{O P T}\right)=\underset{\mu, \alpha}{\arg \max }\{C(\mu, \alpha, \hat{\Delta})\}$,

where $\hat{\Delta}$ is the synchronization phase initially determined. The approach that we use is to determine the optimal synchronization phase when $\mu=v-1$. In this case the interference is null, therefore $\hat{\Delta}=0$ is the optimal time phase [12, 13] and the receiver DFT is applied immediately after the overhead samples.

It is worth noting that with the maximum capacity criterion we optimize not only the $\mathrm{OH}$ parameters and adapt them to a specific channel realization, but we also adapt the set of tones that are switched off to allow the transmitted signal to respect a given PSD mask. We refer to the resulting scheme as adaptive PS-OFDM.

\subsection{Maximum rate criterion}

If we assume to use finite size constellations, under the assumption that the interference is still Gaussian, which is practically the case for a high number of tones [6], we can modify the criterion (16) as follows

$\left(\mu_{O P T}, \alpha_{O P T}\right)=\underset{\mu, \alpha}{\arg \max }\{R(\mu, \alpha, \hat{\Delta})\}$,

where

$$
\begin{aligned}
& R(\mu, \alpha, \hat{\Delta})=\frac{1}{(M+\beta) T} \sum_{k \in K_{O N}(\mu, \alpha)} b^{(k)}(\mu, \alpha, \hat{\Delta}), \\
& b^{(k)}(\mu, \alpha, \hat{\Delta}) \\
& \quad=\left\lfloor\left\lfloor\log _{2}\left(1+\frac{\operatorname{SINR}^{(k)}(\mu, \alpha, \hat{\Delta})}{\Gamma}\right)\right\rfloor .\right.
\end{aligned}
$$

In (19), the operator $\lfloor\lfloor\bullet\rfloor$ denotes the operation of rounding the number of bits to that associated to the nearest available constellation towards zero.

\subsection{Simplified algorithm}

Both the maximization problems in (16) and (17) are complex because they require to compute the set of active tones
$K_{O N}(\mu, \alpha)$ for each pair of values $(\alpha, \mu)$ and the evaluation of the capacity (rate). To simplify the problem we propose to limit the domain of the pair of values $(\alpha, \mu)$. First, we limit the guard interval to the channel length, i.e., $\mu \leq v-1$. Second, simulation results show that if we set $\mu_{M A X}=v-1$, a value $\alpha_{M A X}$ will exist beyond which the cardinality of the set of active tones $K_{O N}$ will not appreciably increase. Consequently, we define the domain of the capacity function as $D=\left\{(\mu, \alpha): 0 \leq \mu \leq \mu_{M A X} \wedge 0 \leq \alpha \leq \alpha_{M A X}\right\}$.

To further reduce the computational complexity, we propose to limit the search of the solution in four sub-domains (search paths) $D_{s} \subset D$ that are defined below. The subdomains identify a certain path within the domain $D$. Figure 3 depicts the four paths.

Path A: The roll-off $\alpha$ is set equal to $\alpha_{M A X}$ while $\mu$ varies between 0 and $\mu_{M A X}$. Therefore, $D_{A}=\{(\mu, \alpha): 0 \leq \mu \leq$ $\mu_{M A X} \wedge \alpha=\alpha_{M A X}$. The pair $(\alpha, \mu)$ given by the solution of (17) is denoted with $\left(\alpha_{O P T, A}, \mu_{O P T, A}\right)$.

Path B: The guard interval $\mu$ is set to $\mu_{M A X}$ and $\alpha$ varies between 0 and $\alpha_{M A X}$. Therefore, $D_{B}=\{(\mu, \alpha): \mu=$ $\left.\mu_{M A X} \wedge 0 \leq \alpha \leq \alpha_{M A X}\right\}$.The pair $(\alpha, \mu)$ given by the solution of (17) is denoted with $\left(\alpha_{O P T, B}, \mu_{O P T, B}\right)$.

Path $C$ : In this case we move along the diagonal of $D$, i.e., $\alpha=\mu$, and we vary $\mu$ between 0 and $\mu_{M A X}$. Therefore, $D_{C}=\left\{(\mu, \alpha): 0 \leq \mu \leq \mu_{M A X} \wedge \alpha=\mu\right\}$. The pair $(\alpha, \mu)$ given by the solution of (17) is denoted with $\left(\alpha_{O P T, C}\right.$, $\left.\mu_{O P T, C}\right)$.

Path D: The guard interval $\mu$ is set to the optimal value $\mu_{O P T, C}$ obtained using path $\mathrm{C}$. Then, $\alpha$ varies from zero to $\alpha_{M A X}$. Therefore, $D_{C}=\left\{(\mu, \alpha): \mu=\mu_{O P T, C} \wedge 0 \leq \alpha=\right.$ (a)

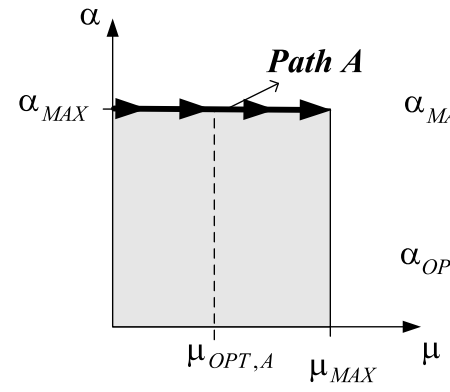

(c)

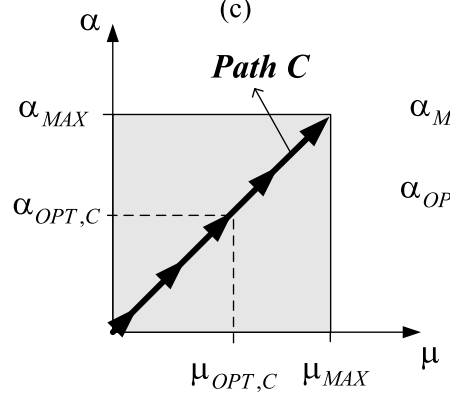

(b)

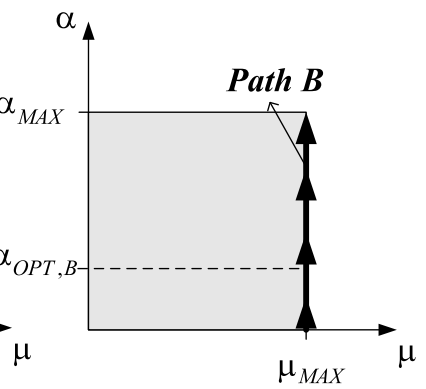

(d)
Fig. 3 Search paths to maximize capacity as a function of $(\mu, \alpha)$ 
$\left.\alpha_{M A X}\right\}$. The pair $(\alpha, \mu)$ given by the solution of (17) is denoted with $\left(\alpha_{O P T, D}, \mu_{O P T, C}\right)$.

\section{Limited adaptation}

The adaptation of the $\mathrm{OH}$ requires that the receiver computes the pair $(\alpha, \mu)$ for each channel realization. A significant simplification will be obtained if the $\mathrm{OH}$ parameters are chosen from a small set of pre-stored values. As an example, the IEEE 1901 standard and the HPAV industry standard [3, 4], [2, Chap. 7] also specify three guard interval lengths (and a single roll-off length).

Herein, we propose an approach to determine the set of values based on the evaluation of the $\mathrm{OH}$ parameters cumulative distribution function (CDF). To obtain the $\mathrm{OH} \mathrm{CDF}$ we apply the statistical PLC channel model from [14] that is also summarized in the next section.

Now, PLC channels can be partitioned into classes according to their capacity [16]. Each class is characterized by an average path loss profile and an average SNR. ${ }^{1}$

For a given channel, the capacity surface as a function of the two $\mathrm{OH}$ parameters (see Sect. 3) exhibits a flat shape around the optimal $\mathrm{OH}$ value (see Sect. 5), i.e, it does not significantly change around the optimal values. Furthermore, for the channels that belong to a given class, the optimal $\mathrm{OH}$ parameters have similar value. In contrast to this, significant changes of the optimal parameter values are found for channels that belong to different classes and that correspond to a notable SNR change. Therefore, we propose to use a unique pair $(\alpha, \mu)$ for all channel realizations that belong to a given average SNR class. Furthermore, we reduce the number of pairs $(\alpha, \mu)$ by considering three values of SNR, i.e., a low, a medium, and a high SNR. This induces a partition of the channels into three classes, only.

For a given average SNR, say $S N R_{\text {low }}$, we determine the joint cumulative distribution function of the optimal pair $(\alpha, \mu)$ (obtained from the realizations of the channel belonging to the given class) that maximizes capacity. We denote with $(\alpha, \mu)_{95 \%}$ the pair that yields a CDF of 0.95 . Then, $(\alpha, \mu)_{95 \%}$ is the $\mathrm{OH}$ pair used for all channel realizations belonging to the given SNR class. If the pair of $\mathrm{OH}$ values for which the $\mathrm{CDF}$ is equal to 0.95 is not unique, we choose that for which the $\mathrm{OH} \beta=\alpha+\mu$ is minimum.

It has to be said that the numerical computation of the joint $\mathrm{CDF}$ is a rather time consuming process. To simplify it, we can limit the computation by considering the sub-domains defined by the paths $A-D$. In this case, since

\footnotetext{
${ }^{1}$ Assuming white additive background noise with PSD $N_{0}$, we can define the average SNR as $\overline{S N R}=\int_{0}^{B} P_{x}(f) P_{L}(f) d f / N_{0} / B$ where $P_{L}(f)=E\left[\left|G_{C H}(f)\right|^{2}\right]$ is the channel average path loss (see (21)).
}

there is only one degree of freedom, the CDF is a function of only one parameter. Thus, we determine $\alpha_{95 \%}$ (SNR) or $\mu_{95 \%}(S N R)$ as the parameter that achieves the 95thpercentile of the CDF.

We emphasize that with this method we limit the amount of adaptation. The algorithm reduces itself to the identification of the channel class and the selection of the pair $(\alpha, \mu)_{95 \%}$ from a look-up table. The identification is done by evaluating the average SNR since in our channel model there is a one-to-one correspondence between the SNR and the channel class. Certainly, the approach of adapting the $\mathrm{CP}$ to the specific channel realization is more robust, and it does not rely on a channel model. However, it is more complex.

\section{Numerical results}

To obtain numerical results we consider the statistical PLC channel model presented in [14]. It synthesizes the channel frequency response with a finite number of multipath components [15] according to

$$
\begin{aligned}
G_{C H}(f) & =A \sum_{i=1}^{N_{P}} g_{i} e^{-\left(a_{0}+a_{1} f^{K}\right) d_{i}} e^{-j 2 \pi f\left(d_{i} / v_{P}\right)}, \\
0 \leq f \leq B . &
\end{aligned}
$$

The number of such components $N_{P}$ is drawn from a Poisson process with average path rate per unit length $\Lambda=$ $0.2 \mathrm{path} / \mathrm{m}$. The maximum path length is $L_{M A X}=800 \mathrm{~m}$. The attenuation factor is denoted with $A$. Further, the reflection factors $g_{i}$ are considered to be uniformly distributed in $[-1,1]$. The parameters have been chosen to fit responses obtained from measurements. They are the following: $K=1, a_{0}=0.3 \times 10^{-2}, a_{1}=4 \times 10^{-10}, v_{P}=2 \times 10^{8}$. The channel has been generated in the band $0-37.5 \mathrm{MHz}$ (see Fig. 2).

As shown in [16], the channel frequency response, at a given frequency, can be approximated with a circularly symmetric complex Gaussian random variable, with amplitude that is Rayleigh distributed. Further, the path loss $P L(f)=E\left[\left|G_{C H}(f)\right|^{2}\right]$ can be computed in closed form as

$$
P_{L}(f)=\frac{A^{2} \Lambda}{3} \frac{1-e^{-2 L_{\max }\left(a_{0}+a_{1} f^{K}\right)}}{\left(1-e^{-\Lambda L_{\max }}\right)\left(2 a_{0}+2 a_{1} f^{K}\right)} .
$$

Figure 4 shows a number of realizations of the channel responses as well as the average path loss.

The signal is transmitted with a power spectral density $P_{x}(f)=P_{x}$ equal to $-50 \mathrm{dBm} / \mathrm{Hz}$ such that the PSD of Fig. 2 is respected. The background noise is assumed to be white Gaussian with a PSD equal to $-140 \mathrm{dBm} / \mathrm{Hz}$ [17]. To obtain three channel classes with different average SNR, the channel is normalized such that the average path loss 


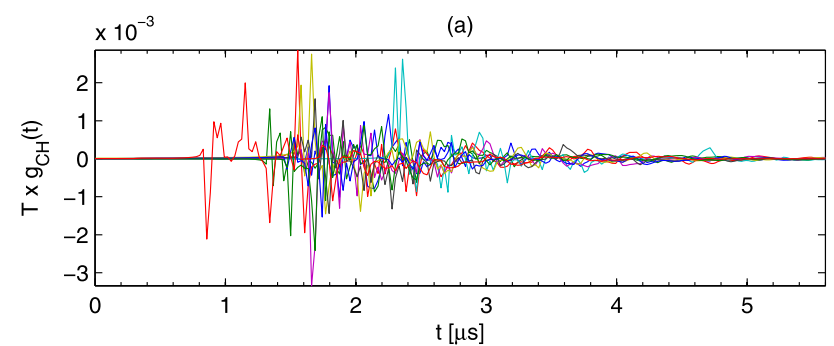

(b)

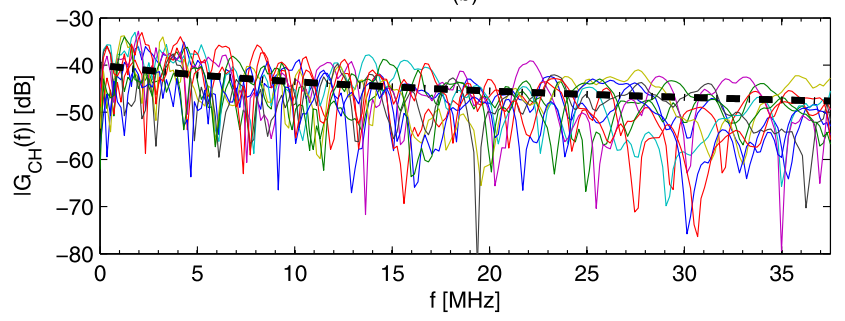

Fig. 4 (a) Ten channel impulse response realizations, and (b) their frequency responses and the average path loss

at zero frequency is equal to 70,50 and $30 \mathrm{~dB}$. Therefore the average SNR at zero frequency ${ }^{2}$ is respectively equal to 20,40 , and $60 \mathrm{~dB}$. The average SNR at zero frequency is used to label the performance curves in the numerical results section. The average $\overline{S N R}$ (see Footnote 1) is respectively equal to $16.7 \mathrm{~dB}, 36.7 \mathrm{~dB}$ and $56.7 \mathrm{~dB}$.

For the transmitter, we choose parameters similar to those employed in HPAV [18]. The number of sub-channels is $M=1536$ in the band $0-37.5 \mathrm{MHz}$. The transmission window is either rectangular or raised cosine. The $\mathrm{OH}$ parameters can assume the maximum values $\mu_{M A X}=\alpha_{M A X}=$ $5.6 \mu \mathrm{s}$. The QAM constellations that we deploy for bitloading have size $2^{c}$ with $c=[1,2,4,6,8,10]$. The gap factor is set to $\Gamma=9 \mathrm{~dB}$.

Finally, we let the parameters $\mu$ and $\alpha$ vary in steps of 10 samples $(0.267 \mu \mathrm{s})$.

\subsection{Performance with adaptation}

As discussed, both $\mu$ and $\alpha$ have an effect on the subchannel frequency response in PS-OFDM. In turn, this affects the number of tones that need to be switched off to fulfill a certain notching mask. Let us consider the notching mask of Fig. 2. Although it is not shown, for the path $A$, where we set the roll-off equal to $\alpha=5.6 \mu$ s, the increase of the guard interval does not appreciably increase the number of active sub-channels. In other words, the guard interval does not significantly affect the signal PSD once the roll-off is set to $5.6 \mu \mathrm{s}$. In contrast to this, for the other paths the number of active sub-channels increases significantly by increasing the roll-off factor up to $\alpha_{M A X}$. For all the paths, the maximum number of active tones is equal to 827 .

\footnotetext{
${ }^{2}$ Assuming a constant transmitted PSD $P_{x}$, the average SNR at zero frequency can be defined as $S N R=P_{x} P_{L}(0) / N_{0}$.
}

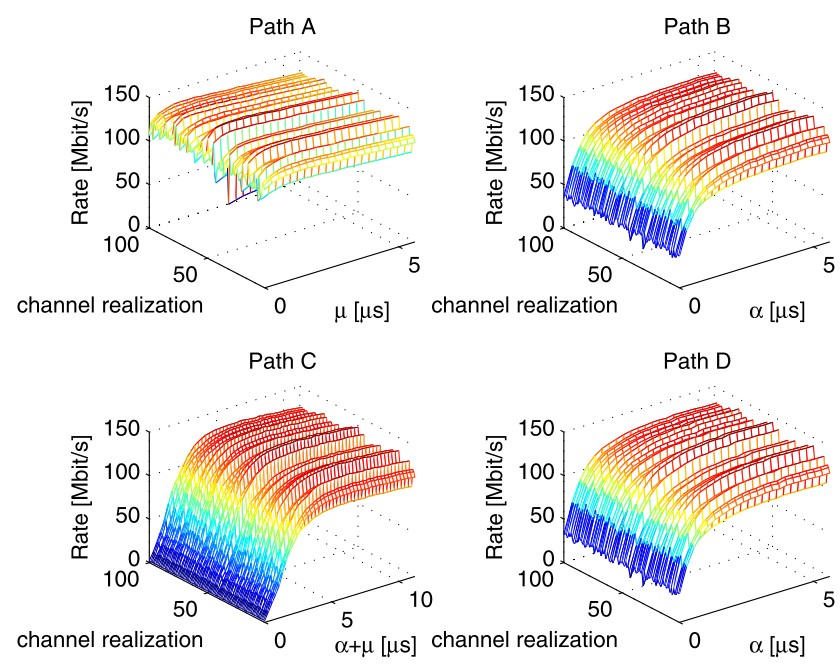

Fig. 5 Bit-rate as a function of overhead parameters restricted to the search paths in Fig. 3 for 100 channel realizations. The SNR is equal to $40 \mathrm{~dB}$

In Fig. 5, we show the bit-rate (18) that is obtained when the search of the $\mathrm{OH}$ parameters is done moving through the four paths in Fig. 3 for an SNR equal to $40 \mathrm{~dB}$.

We observe that an optimal pair $(\alpha, \mu)$ can be found for each of the 100 channel realization herein considered. The rate-optimal $\mathrm{OH}$ values depend on the search path, and the achievable-rate varies significantly as function of $(\alpha, \mu)$.

To determine the achievable gains, we deploy, as a baseline, the system that uses a constant value for both the guard interval and the roll factor that are equal to the channel length of $5.6 \mu$ s. Figure 6 shows the bit-rate gains obtained adapting the GI and the RO to the channel condition using the rate maximization criterion (17) along the four paths. The gains are computed w.r.t. to the baseline system that uses a constant $\mathrm{OH}$. The gains are reported for 100 channel realizations assuming an SNR equal to $20 \mathrm{~dB}, 40 \mathrm{~dB}$ and $60 \mathrm{~dB}$. As we can see, path $A$ gives the best results for all SNR cases and for all channel realizations. For this path the gains range between $5 \%$ and $11 \%$.

It is interesting to note that high gains are obtained for low SNRs. This can be explained by observing that for low SNRs the noise dominates the interference. Thus, in the rate formula (18), the rate loss given by the factor $M /(M+\beta)$ outweighs the rate gain from the SINR increase due to an increase of the $\mathrm{OH}$ and in particular of the GI.

In Table 1, we report the average bit-rate that is obtained with the rate maximization criterion with the search path $A$, and the bit-rate that we will obtain if we set both the GI and the RO equal to $5.6 \mu$ s (baseline system). Table 1 also lists the bit-rate obtained using limited adaptation as discussed in the next subsection.

Now, in Fig. 7, we show the bit-rate that is achieved when no notching is required. A comparison with OFDM is also reported. The figure shows that the rate curves have a 
(a): $S N R=20 d B$

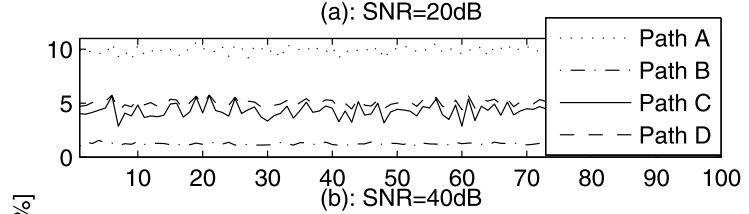

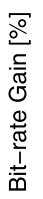
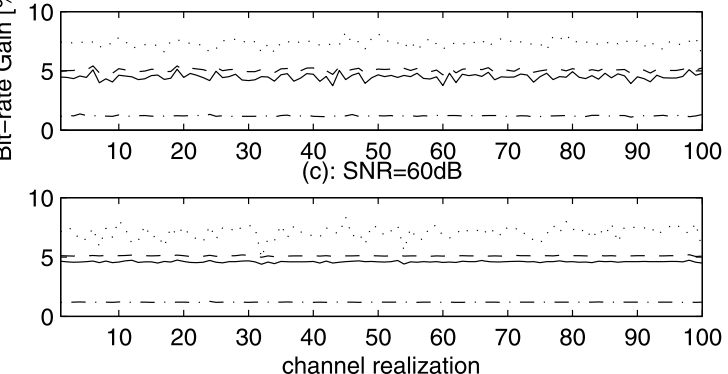

Fig. 6 Bit-rate gain given by the adaptation of the GI and of the RO for 100 channel realizations obtained with the rate maximization criterion along the four paths in Fig. 3. The SNR has been set to (a): $20 \mathrm{~dB}$, (b): $40 \mathrm{~dB}$, and (c): $60 \mathrm{~dB}$. The gains have been computed w.r.t. the bit-rate obtained when using the constant values $\mu=5.6 \mu$ s

Table 1 Average bit-rate comparisons: masked case

\begin{tabular}{|c|c|c|c|}
\hline & & PS-OFDM & \\
\hline $\begin{array}{l}\text { SNR } \\
{[\mathrm{dB}]}\end{array}$ & $\begin{array}{l}\text { Avg. rate } \\
{[\mathrm{Mbit} / \mathrm{s}]} \\
\alpha=5.6 \mu \mathrm{s} \\
\mu \text {-adap. }\end{array}$ & $\begin{array}{l}\text { Avg. rate } \\
{[\mathrm{Mbit} / \mathrm{s}]} \\
\alpha=5.6 \mu \mathrm{s}\end{array}$ & $\begin{array}{l}\text { Avg. rate } \\
{[\mathrm{Mbit} / \mathrm{s}]} \\
\alpha=5.6 \mu \mathrm{s} \\
\mu=5.6 \mu \mathrm{s}\end{array}$ \\
\hline 20 & 34.7 & $\begin{array}{l}34.7 \\
\mu_{95 \%}=0.25 \mu \mathrm{s}\end{array}$ & 31.5 \\
\hline 40 & 126.3 & $\begin{array}{l}126.2 \\
\mu_{95 \%}=1.6 \mu \mathrm{s}\end{array}$ & 117.7 \\
\hline 60 & 169 & $\begin{array}{l}168 \\
\mu_{95 \%}=2 \mu \mathrm{s}\end{array}$ & 157.8 \\
\hline
\end{tabular}

more pronounced convex shape than those in Fig. 5. OFDM achieves a higher rate than PS-OFDM that uses a fixed $\alpha=5.6 \mu$ s. However, it should be noted that if we adapted the roll-off factor, then PS-OFDM would use a roll-off equal to zero, thus, falling back to OFDM. However, although not shown here, we note that OFDM has poor performance when notching is required.

Finally, Table 2 summarizes the results in terms of average bit-rate for OFDM and PS-OFDM without notching.

\subsection{Limited adaptation}

As discussed in the previous subsection, solving (17) along path $A$ gives the best results for the channels and notching mask that we have considered. Nevertheless, it is worth noting that the bit-rate curves (Fig. 5) show that the bit-rate will not dramatically change if we choose an $\mathrm{OH}$ close but not exactly identical to the optimal one. This observation (a): UNMASKED OFDM

(b): UNMASKED PS-OFDM Path A
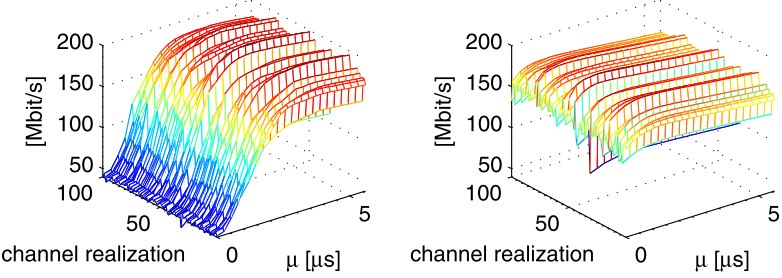

Fig. 7 Bit-rate for the Unmasked case using the search path A. (a): OFDM. (b): PS-OFDM. The SNR is equal to $40 \mathrm{~dB}$

Table 2 Average bit-rate comparisons: unmasked case

\begin{tabular}{|c|c|c|c|c|}
\hline \multirow{5}{*}{$\begin{array}{l}\text { SNR } \\
\text { [dB] }\end{array}$} & \multicolumn{2}{|l|}{ OFDM } & \multicolumn{2}{|l|}{ PS-OFDM } \\
\hline & Avg. rate & Avg. rate & Avg. rate & Avg. rate \\
\hline & [Mbit/s] & [Mbit/s] & [Mbit/s] & [Mbit/s] \\
\hline & $\alpha=0$ & $\alpha=0$ & $\alpha=5.6 \mu \mathrm{s}$ & $\alpha=5.6 \mu \mathrm{s}$ \\
\hline & $\mu$-adap. & $\mu=5.56 \mu \mathrm{s}$ & $\mu$-adap. & $\mu=5.6 \mu \mathrm{s}$ \\
\hline 20 & 49.3 & 46.1 & 45.7 & 41.2 \\
\hline 40 & 176.1 & 170.6 & 164.1 & 152.3 \\
\hline 60 & 234.4 & 227.7 & 218.1 & 203.2 \\
\hline
\end{tabular}

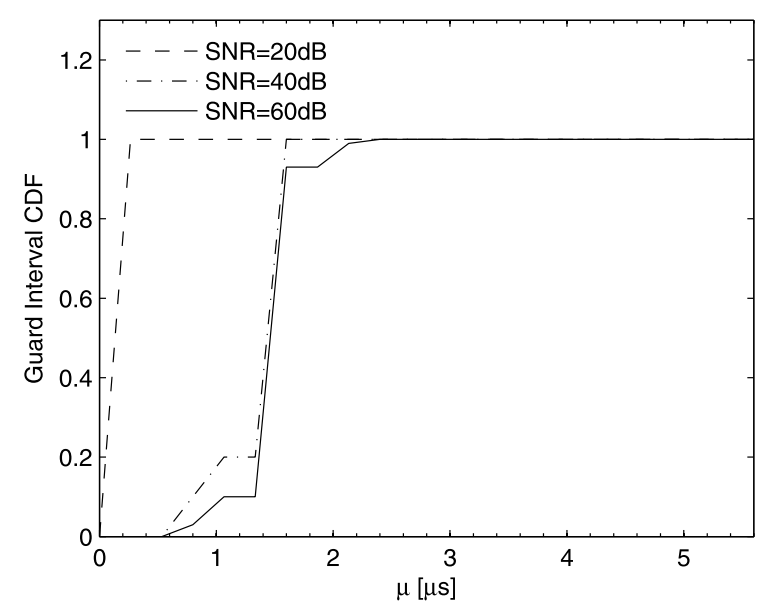

Fig. 8 Guard interval CDF using the search path A

justifies the idea of limiting the adaptation, in particular the idea of using the $\mathrm{OH} \mathrm{CDF}$ to find the finite set of $\mathrm{OHs}$ as described in Sect. 4.

In Fig. 8, we show the guard interval CDF obtained solving (17) when the search is done along path $A$. The guard intervals that yield a CDF equal to 0.95 are: $\mu_{95 \%}(20 \mathrm{~dB})=$ $0.25 \mu \mathrm{s}, \mu_{95 \%}(40 \mathrm{~dB})=1.6 \mu \mathrm{s}, \mu_{95 \%}(60 \mathrm{~dB})=2 \mu \mathrm{s}$. Recalling that the roll-off for path $A$ is equal to $\alpha_{O P T, A}=$ $5.6 \mu \mathrm{s}$, we can compute the $\mathrm{OH}$ values as $\beta(S N R)=$ $\alpha_{O P T, A}+\mu_{95 \%}(S N R)$. They are equal to $\beta(20 \mathrm{~dB})=5.85 \mu \mathrm{s}$, $\beta(40 \mathrm{~dB})=7.2 \mu \mathrm{s}$, and $\beta(60 \mathrm{~dB})=7.6 \mu \mathrm{s}$.

HPAV specifies a very large value of $\mathrm{OH}$ that is used for the synchronization channel, and two $\mathrm{OH}$ values that are used during normal transmission [2]. The latter two $\mathrm{OH}$ val- 
ues are equal to $5.56 \mu$ s, and $7.56 \mu$ s. Further, the RO is set to $4.96 \mu$ s. These values are close to the ones that we have computed with the proposed method. The roll-off duration of HPAV is smaller than the one we obtained. It has however to be said that HPAV uses a piece wise linear window [19]. Although not reported here, simulation results show that using the raised cosine window with a roll-off equal to $4.96 \mu$ s, the bit rate obtained along path $A$ is smaller than the one obtained with a roll-off equal to $5.6 \mu$ s. This is because the number of active tones with roll-off equal to $4.96 \mu$ s is notably smaller than that with a roll-off of $5.6 \mu$ s using the raised cosine window.

Finally, in Table 1 and 2 we summarize the performance results in terms of average bit-rate. The main remarks are the following:

1. When notching is not required, the optimal roll-off is equal to zero, i.e., PS-OFDM becomes identical to OFDM. In such a case the average gains obtained by adapting the GI w.r.t. to using a constant GI as long as the channel, span between $3 \%$ and $7 \%$;

2. The search path A yields the highest bit-rate among the four search paths considered, when we use the HPAV notching mask of Fig. 2. In such a case the roll-off factor is equal to $5.6 \mu \mathrm{s}$ and the adaptation of the GI yields gains, w.r.t. a GI equal to the channel length, that range between $5 \%$ and $11 \%$;

3. The limited adaptation of the GI based on the CDF criterion gives an average bit-rate very close to the one that we achieve if we fully adapt the GI.

\section{Conclusions}

We have proposed an adaptive pulse-shaped OFDM scheme where the $\mathrm{OH}$ parameters (guard interval and roll-off factor) are adapted to jointly maximize capacity and fulfill a desired notching mask. The adaptation can be done when the channel response and/or the notching mask changes. This realizes a form of cognitive PLC system if the notching mask is adapted according to the presence or absence of interferers in the transmission spectrum.

To lower the computational cost, we have also proposed to limit the amount of adaptation, i.e., to use a finite set of $\mathrm{OH}$ parameters. The $\mathrm{OH}$ values can be computed from the analysis of their distribution function in statistically representative channels. Since a significant change of the $\mathrm{OH}$ parameters is caused by a significant change in the SNR, we have considered the design of the $\mathrm{OH}$ parameters for a low, a medium, and a high SNR scenario assuming a certain target notching mask. Thus, the adaptation is limited to the computation of the SNR at the receiver, and feedback to the transmitter of the best $\mathrm{OH}$ parameters for such an SNR.
Several performance results show that both the full adaptation and the limited adaptation provide notable gains compared to the case of using constant suboptimal $\mathrm{OH}$ parameters, i.e., an RO that minimizes the number of switched off tones and a GI as long as the channel.

Open Access This article is distributed under the terms of the Creative Commons Attribution Noncommercial License which permits any noncommercial use, distribution, and reproduction in any medium, provided the original author(s) and source are credited.

\section{Appendix: Derivation of the useful and the interference power}

The PS-OFDM is a DFT modulated filter bank system with Nyquist prototype synthesis $g(n)$, and analysis $h(n)$ pulses as defined in (6), (7). The signal at the output of the $i$-th analysis pulse (or equivalently the $i$-th DFT output) at time instant $\ell N$, in the absence of noise and with the channel model in (2), can be written as

$$
\begin{aligned}
z_{\text {signal }}^{(i)}(\ell N+\Delta)= & \sum_{m \in \mathbb{Z}} \sum_{p=0}^{v-1} \sum_{k \in K_{O N}} \alpha_{p} a^{(k)}(m N) \\
& \times r_{g h}^{(k, i)}(\mu, \alpha, \ell N-m N+\Delta-p) \\
& \times e^{j 2 \pi \frac{k \ell N}{M}} e^{-j 2 \pi \frac{i \ell N+\Delta)}{M}}
\end{aligned}
$$

with

$r_{g h}^{(k, i)}(\mu, \alpha, n)=g^{(k)}(n) * h^{(i)}(n)$,

and

$g^{(k)}(n)=g(n) e^{j 2 \pi \frac{k n}{M}}, \quad h^{(i)}(n)=h(n) e^{j 2 \pi \frac{i n}{M}}$.

In (22), $\Delta$ denotes the synchronization phase. In (23) we have explicitly indicated the dependence of the crosstalk impulse response term $r_{g h}^{(k, i)}(\mu, \alpha, n)$ at the instant $n$ on the parameters $\mu$ and $\alpha$.

The useful signal term can be obtained setting in (22) $k=i$ and $m=\ell$, i.e.,

$$
\begin{aligned}
z_{U}^{(i)}(\ell N+\Delta)= & a^{(i)}(\ell N) \sum_{p=0}^{v-1} \alpha_{p} r_{g h}^{(i, i)}(\mu, \alpha, \Delta-p) \\
& \times e^{-j 2 \pi \frac{i \Delta}{M}} \\
= & a^{(k)}(\ell N) H^{(k)}(\mu, \alpha, \Delta) .
\end{aligned}
$$

The interference term $I^{(k)}(\mu, \alpha, \ell N+\Delta)$ can be computed setting in (22) $k \neq i$ and $m \neq \ell$.

The total power of the received sub-channel signal (22), i.e., $P_{T O T}^{(i)}(\mu, \alpha, \Delta)=E\left[\left|z_{\text {signal }}^{(i)}(\ell N+\Delta)\right|^{2}\right]$, depends on the $\mathrm{OH}$ parameters and on $\Delta$. It can be computed assuming the 
data symbols to be independent, with zero mean and power $P_{a}$. The calculation yields:

$$
\begin{aligned}
P_{T O T}^{(i)}(\mu, \alpha, \Delta)= & P_{a} \sum_{\ell \in \mathbb{Z}} \sum_{k \in K_{O N}} \sum_{p, p^{\prime}=0}^{\nu-1} \alpha_{p}\left(\alpha_{p^{\prime}}\right)^{*} \\
& \times r_{g h}^{(k, i)}(\mu, \alpha, \ell N-m N+\Delta-p) \\
& \times\left(r_{g h}^{(k, i)}\left(\mu, \alpha, \ell N-m N+\Delta-p^{\prime}\right)\right)^{*},
\end{aligned}
$$

where $(\cdot)^{*}$ denotes the complex conjugate operator.

If we set in (26), $k=i$ and $m=\ell$, we obtain the power of the useful received signal in the $k$-th sub-channel, i.e.,

$$
\begin{aligned}
P_{U}^{(k)}(\mu, \alpha, \Delta)= & P_{a} \sum_{p, p^{\prime}=0}^{\nu-1} \alpha_{p}\left(\alpha_{p^{\prime}}\right)^{*} r_{g h}^{(k, k)}(\mu, \alpha, \Delta-p) \\
& \times\left(r_{g h}^{(k, k)}\left(\mu, \alpha, \Delta-p^{\prime}\right)\right)^{*} .
\end{aligned}
$$

The interference power (12) can be computed as the difference between the total and the useful power.

\section{References}

1. Weinstein, S. B., \& Ebert, P. M. (1971). Data transmission by frequency-division multiplexing using the discrete Fourier transform. IEEE Transactions on Communication Technology, 9(5).

2. Ferreira, H., Lampe, L., Newbury, J., \& Swart, T. (Eds.) (2010). Power line communications. New York: Wiley.

3. Galli, S., \& Logvinov, O. (2008). Recent developments in the standardization of power line communications within the IEEE. IEEE Communications Magazine 46(7), 64-71.

4. Latchman, H., Afkhamie, K., Katar, S., Mashburn, B., Newman, R., \& Yonge, L. (2005). High speed multimedia home networking over powerline. In NCTA technical papers (pp. 9-22).

5. Peled, A., \& Ruiz, A. (1980). Frequency domain data transmission using reduced computational complexity algorithms. In Proc. of IEEE int. conf. on acoustic, speech and signal processing (ICASSP) 1980 (Vol. 5, pp. 964-967).

6. Seoane, J., Wilson, S., \& Gelfand, S. (1995). Analysis of intertone and interblock interference in OFDM when the length of the cyclic prefix is shorter than the length of the impulse response of the channel. In Proc. of IEEE global telecommun. conf. (GLOBECOM) (pp. 2069-2074), Singapore.

7. Tonello, A. M., D'Alessandro, S., \& Lampe, L. (2010). Cyclic prefix design and allocation in bit-loaded OFDM over power line communication channels. IEEE Transactions on Communications, $58(11)$.

8. Kalet, I. (1989). The multitone channel. IEEE Transactions on Communications, 37(2).

9. Sjoberg, F., Nilsson, R., Isaksson, M., Odling, P., \& Borjesson, P. O. (1999). Asynchronous zipper. In IEEE int. conf. on commun. (ICC) (Vol. 1, pp. 231-235).

10. Proakis, J. G. (1995). Digital communications (3th ed.). New York: McGraw-Hill.

11. Cioffi, J. M. Chapter 4, Advanced digital communication. Available at http://www.stanford.edu/class/ee379c/reader.html.
12. Schmidl, T. M., \& Cox, D. C. (1997). Robust frequency and time synchronization for OFDM. IEEE Transactions on Communications, 45(12).

13. Pollet, T., \& Peeters, M. (1999). Synchronization with DMT modulation. IEEE Communications Magazine, 37(4), 80-86.

14. Tonello, A. M. (2007). Wideband impulse modulation and receiver algorithms for multiuser power line communications, EURASIP Journal on Advances in Signal Processing, 2007, 96747. doi:10.1155/2007/96747, 14 pages.

15. Zimmermann, M., \& Dostert, K. (2002). A multipath model for the powerline channel. IEEE Transactions on Communications, 50, 553-559.

16. Pagani, P., Tlich, M., Zeddam, A., Tonello, A., Pecile, F., D’Alessandro, S., Mijic, G., \& Kriznar, K. (2009). PLC channel transfer function models for the OMEGA ICT project. In: ICTMobile Summit 2009, Santander.

17. Cañete, F., Cortés, J., Díez, L., \& Entrambasaguas, J. (2006). Analysis of the cyclic short-term variation of indoor power line channels. IEEE Journal on Selected Areas in Communications, 24(7), 1327-1338.

18. Home Plug AV White Paper, Version HPAVWP-050818 (2005).

19. Afkhamie, H., Latchman, H., Yonge, L., Davidson, T., \& Newman, R. (2005). Joint optimization of transmit pulse shaping, guard interval length, and receiver side narrow-band interference mitigation in the HomePlugAV OFDM system. In IEEE int. workshop on signal processing advances for wireless commun. (SPAWC) 2005, New York.

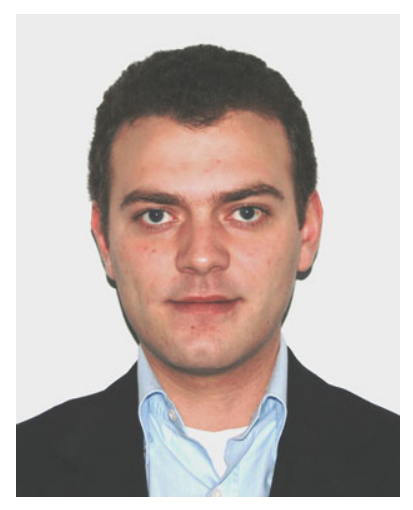

Salvatore D'Alessandro received the Laurea and the Magistrale degrees in electrical engineering from the University of Udine, Italy, in 2006 and 2008, respectively. In 2007 he was a visiting student for a semester at the University of Málaga (Spain) working on power line communications. Currently, he is a Ph.D. student at the University of Udine. His research activity focuses on power line communications, resource allocation algorithms, signal processing for communications.

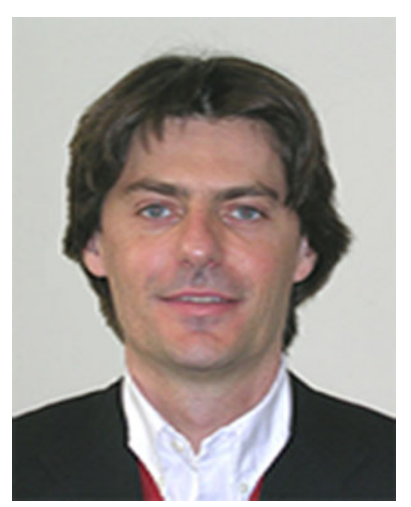

Andrea M. Tonello received the Doctor of Engineering degree in electronics (cum laude), and the Doctor of Research degree in electronics and telecommunications, both from the University of Padova, Italy. On February 1997, he joined as a Member of Technical Staff, Bell Labs-Lucent Technologies, where he worked on the development of baseband algorithms for cellular handsets, first in Holmdel, $\mathrm{NJ}$, and then within the Philips/ Lucent Consumer Products Division in Piscataway, NJ. From September 1997 to December 2002, he has been with the Bell Labs Advanced Wireless Technology Laboratory, Whippany, NJ. He was promoted in 2002 to Technical Manager, and was appointed Managing Director of Bell Labs, Italy. In January 2003, he joined the Dipartimento di Ingegneria Elettrica, Gestionale, e Meccanica (DIEGM) of the University of Udine, Italy, where he is an Aggregate Professor. 
He received a Lucent Bell Labs Recognition of Excellence award for his work on enhanced receiver techniques in 2003, the award for the best paper published in Eurasip Journal on Advances in Signal Processing about ultra wide band PLC communication 2007, and he is the coauthor of the best student paper award at the IEEE International Symposium on Power Line Communications (ISPLC) 2010. He was co-recipient of two awards in the business plan competition Startcup 2007 and 2008 in Udine, Italy. He was awarded a Distinguished Visiting Fellowship from the Royal Academy of Engineering, UK, in 2010. He was the TPC Co-chair of IEEE ISPLC 2007, the Chair of the Workshop on Power Line Communications 2009, and the General Chair of IEEE ISPLC 2011. He serves as an Associate Editor for the IEEE Transactions on Vehicular Technology. He is the Vice-chair of the IEEE Communications Society Technical Committee on Power Line Communications.

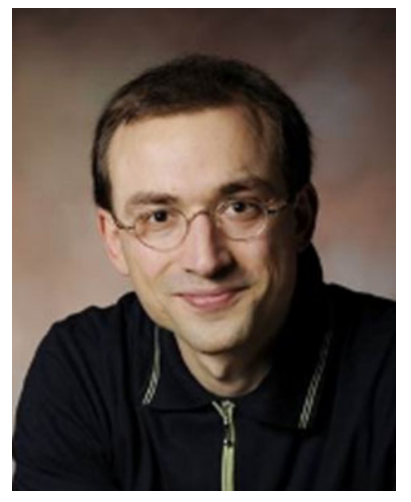

Lutz Lampe received the Diplom (Univ.) and the Ph.D. degrees in electrical engineering from the University of Erlangen, Germany, in 1998 and 2002, respectively. Since 2003 he has been with the Department of Electrical and Computer Engineering at the University of British Columbia, where he is a Full Professor.

$\mathrm{He}$ is co-recipient of the Eurasip Signal Processing Journal Best Paper Award 2005 and Best Paper Awards at the 2006 IEEE International Conference on Ultra-

Wideband (ICUWB) and the 2010 IEEE International Conference on Communications (ICC). In 2003, he received the Dissertation Award of the German Society of Information Techniques (ITG). He was awarded the UBC Killam Research Prize in 2008, the Friedrich Wilhelm Bessel Research Award by the Alexander von Humboldt Foundation in 2009, and the UBC Charles A. McDowell Award of Excellence in Research in 2010 .

$\mathrm{He}$ is an Editor for the IEEE Transactions on Wireless Communications and the International Journal on Electronics and Communications (AEUE). He was the General Chair of the 2005 International Symposium on Power Line Communications (ISPLC) and the 2009 IEEE International Conference on Ultra-Wideband (ICUWB). He is the Chair of the IEEE Communications Society Technical Committee on Power Line Communications. 